A.M. Steinberg, R. Sadanandan, C. Dem, P. Kutne, W. Meier, Structure and stabilization of hydrogen jet flames in cross-flows, Proc. Combust. Inst. 34 (2013) 1499-1507

The original publication is available at www.elsevier.com

http://dx.doi.org/10.1016/j.proci.2012.06.026 


\title{
Structure and stabilization of hydrogen jet flames in cross-flows
}

\author{
A. M. Steinberg ${ }^{a}$, R. Sadanandan ${ }^{b}$, C. Dem ${ }^{b}$, P. Kutne ${ }^{b}$ and W. Meier ${ }^{b}$ \\ ${ }^{a}$ Institute for Aerospace Studies, University of Toronto, Toronto M3H 5T6, Canada \\ ${ }^{b}$ Institut für Verbrennungstechnik, Deutsches Zentrum für Luft- und Raumfahrt (DLR), 70569 Stuttgart, Germany
}

\begin{abstract}
The structure and stabilization of heated hydrogen jet flames in heated cross-flows was experimentally investigated in a configuration that is analogous to terrestrial gas turbine components. Three flames, with jet velocities ranging from 100$200 \mathrm{~m} / \mathrm{s}$, were investigated using particle image velocimetry and $\mathrm{OH}$ planar laser induced fluorescence in a total of $11 x-y$ and $y-z$ planes. Additionally, laser Raman scattering was performed in the $200 \mathrm{~m} / \mathrm{s}$ jet to characterize the thermo-chemical state. In all cases, the flame along the jet centerline plane consisted of two branches, one stabilized in the jet lee and one lifted above the jet trajectory. The positional stability of the lee-stabilized branch was greater in the higher jet velocity cases due to the larger and stronger recirculation zones created downstream of the injection point. The lifted flame branch was much more dynamic, with measured flame base axial positions ranging from the jet near field to the flame tip. This flame branch instantaneously resided downstream of regions with high extensive principal strain-rate, and the strain-rate significantly affected the thermo-chemical state. The Raman measurements indicated that the base of the lifted flame branch existed in locations where both tribrachial and/or stratified premixed flame behaviors are expected, depending on the instantaneous flame location. Accurately modeling these complex flame structures and flow-flame interactions therefore is necessary to properly simulate jet flames in cross-flows.
\end{abstract}

Keywords: Jet in cross-flow; flame stabilization; tribrachial flames; partially premixed flames; hydrogen 


\section{Introduction}

This paper investigates the structure and stabilization of jet flames in cross-flows. The jet-in-cross-flow (JICF) is a geometrically simple configuration, but one that possesses an array of complex features and finds numerous practical applications [1]. In combustion systems, JICFs are used to enhance the mixing of fuel and oxidizer. For example, aerospace gas turbine engines employ JICFs for injection of secondary fuel or dilution air [2, 3], while certain terrestrial gas turbines use them in combustion staging schemes for ultra-low $\mathrm{NO}_{x}[4]$. Fuel injectors for high-speed cross-flows, such as those occurring in ramand scramjet engines, also often utilize JICFs [5-7].

Regardless of the application, the ability or inability of a flame to stabilize in the vicinity of the jet is of critical importance. In the case of staged gas-turbines, reheat fuel must not burn in the jet wake, but mix thoroughly with hot primary-exhaust air before burning further downstream. This keeps peak combustion temperatures low and reduces $\mathrm{NO}_{x}$ production. However, in other gas turbine configurations and high-speed propulsion devices, flames should occur relatively near to the jet, and the stabilization position is an important parameter.

This work experimentally investigates the relationship between the flow field, mixing, and flame stabilization for three hydrogen/air JICF flames. The configuration and conditions are designed to provide a simplified analog for components found in practical gas turbine combustors, particularly those being investigated for low $\mathrm{NO}_{x}$ terrestrial power generation using alternative fuels [4]. Similar conditions are currently being studied using direct numerical simulations (DNS) [8,9].

In general, a JICF flow field consists of several vortical systems. The largest and most widely studied of these is the kidneyshaped counter-rotating vortex pair (CVP) that aligns roughly parallel the jet trajectory, starting some distance downstream from the injection location [1]. These structures enhance mixing, and therefore greatly influence the downstream behavior of the flame. Experimental and computational studies indicate that the CVP originates due to distortion of vorticity in shear layer between the jet and cross-flow [10-12]. DNS simulations also highlight the importance of the pressure difference between the windward and leeward edges of the jet in generating sufficient vorticity to form the CVP downstream [13, 14].

In addition to the CVP, JICFs issuing flush from a wall exhibit vortical systems in the form of horseshoe vortices and downstream wake vortices. The horseshoe vortices wrap around the jet base from the windward side in the boundary layer, arise from the displacement of the cross-flow boundary layer by the jet, and may exhibit both stationary and oscillatory modes

[10]. Wake vortices are upright structures that connect the boundary layer to downstream regions of the jet. These structures promote entrainment of boundary layer fluid into the jet wake, enhancing mixing [15].

However, while these flow structures are important for all JICFs, they are not necessarily intrinsic to flame stabilization. Relatively few studies have addressed the near-field flame structure, the interaction between this flame and the flow, or how this interaction influences flame stabilization [1]. Huang et al. [16] identify five different flame and flow modes for JICFs, depending on the jet-to-cross-flow momentum flux ratio

$$
r=\left(\rho_{j} u_{j}^{2} / \rho_{c} u_{c}^{2}\right)^{1 / 2}
$$

where $u_{j}$ and $u_{c}$ are the jet and cross-flow bulk velocities, respectively. However, for jets issuing flush from a wall, only two flame modes are relevant. These are referred to alternatively as the 'burner attached' and 'burner detached' or 'lifted' and 'wake-stabilized' modes. The remainder of this paper will investigate these different flame modes in order to better understand 


\begin{tabular}{cccc}
\hline \hline Case & $\begin{array}{c}u_{j} \\
(\mathrm{~m} / \mathrm{s})\end{array}$ & $r$ & $\begin{array}{c}R e_{j}= \\
u_{j} D_{j} / \nu_{j}\end{array}$ \\
\hline 1 & 100 & 1.4 & 3000 \\
2 & 150 & 2.2 & 4500 \\
3 & 200 & 2.9 & 6000 \\
\hline \hline \multicolumn{4}{c}{ Table 1: Jet conditions. }
\end{tabular}

the structure and stabilization of jet flames in cross-flows.

\section{Experiment and diagnostics}

\subsection{Jet-in-crossflow facility}

An optically accessible flow channel with a $40 \mathrm{~mm}$ x $60 \mathrm{~mm}$ rectangular cross section was used for the experiments (Fig. 1). The channel consisted of a settling chamber, a modular optically accessible section that included the fuel injection module, and an exhaust section. Each optical module was composed of four fused silica windows held together by stainless steel corner posts and end plates. At the fuel injection module, one of the $40 \mathrm{~mm}$ wide windows was replaced with a stainless steel plate containing the injector. The diameter of the circular fuel injector tube $\left(D_{j}=2 \mathrm{~mm}\right)$ was kept constant for at least $20 D_{j}$ to ensure a well developed flow field. The entire flow channel was mounted on a three-axis translation stage, which allowed precise positioning of the desired measurement locations relative to the laser diagnostics. No contraction or end plate was used at the duct exhaust and coherent pressure oscillations were minimal.

Electrically preheated fuel $\left(\mathrm{H}_{2} / \mathrm{N}_{2}\right.$ at $70 / 30$ vol. $\left.\%, T_{j}=423 \mathrm{~K}\right)$ was injected into a cross flow of electrically preheated air $\left(T_{c}=750 \mathrm{~K}\right)$ at an angle of 90 degrees. The air flow rate was set such that the bulk flow velocity was $u_{c}=55 \mathrm{~m} / \mathrm{s}$. However, the centerline velocity (with zero fuel injection) was approximately $59 \mathrm{~m} / \mathrm{s}$ due to boundary layers. Three different fuel flow rates were studied, which are described in Table 1 . These cases and conditions are similar to those used in wider study that was carried out under the European project BIGH2. The detailed measurements presented here were carried out only on this targeted subset. Case $3\left(u_{j}=200 \mathrm{~m} / \mathrm{s}\right)$ is the maximum injection velocity at which a flame could be stabilized under these conditions.

An important flow characteristic for validation of numerical simulations is the boundary layer thickness. Hence, the crossflow boundary layer at the injection location was characterized using particle image velocimetry (described below), with the injection block replaced by a fused silica window. Over the measurement region, the boundary layer velocity thickness, defined at $u_{x}=0.99 u_{c}$, was essentially constant at $2 \mathrm{~mm}$.

\subsection{Diagnostics}

Three different experiments were conducted. In the first experiment (E1, shown in Fig. 1), simultaneous OH planar laser induced fluorescence (PLIF) and stereoscopic particle image velocimetry (S-PIV) were conducted in five $x-y$ planes at $z=\{0,1,2,5,10\} \mathrm{mm}$. In the second experiment (E2, setup not shown), OH PLIF and two-component PIV were conducted in six $x-z$ planes at $y=\{3,6,9,12,15,18\} \mathrm{mm}$. S-PIV could not be conducted in this orientation due to optical access restrictions. It is noted that not all planes will be utilized in the present discussion. Finally, laser Raman scattering measurements (E3, setup not shown) were conducted at selected points in the flow for Case 3.

The OH-PLIF system consisted of a frequency doubled Nd:YAG laser (Quanta Ray, DCR-2) pumping a frequency doubled tunable dye laser (Lumonics, HD-500). The dye laser output at approximately $283 \mathrm{~nm}$ (pulse duration $8 \mathrm{~ns}$, pulse energy 3 mJ, 


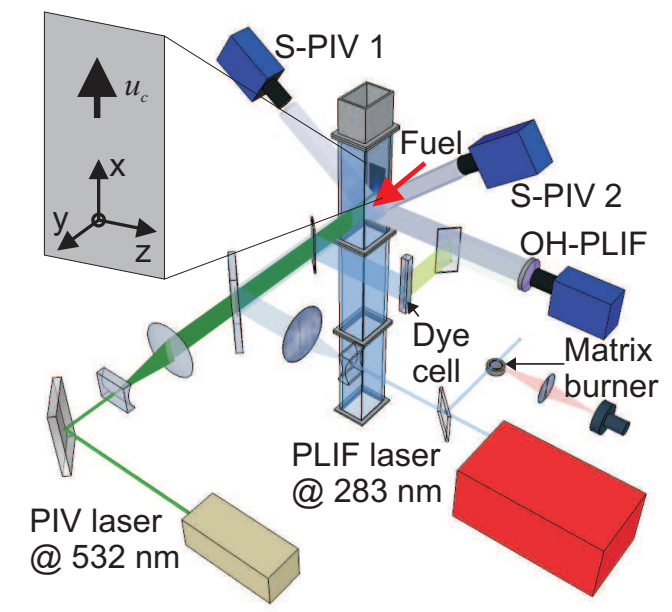

Fig. 1: Experimental setup for the simultaneous stereoscopic PIV and OH PLIF system used for measurements in the $x-y$ plane during experiment E1.

line width $\approx 0.4 \mathrm{~cm}^{-1}$ ) was tuned to the $\mathrm{Q}_{1}(8)$ transition of $\mathrm{OH}$ in the $\nu^{\prime \prime}=0, \nu^{\prime}=1$ vibrational band of the $A^{2} \Sigma-X^{2} \Pi$ system. A small portion of this laser beam was directed into a laminar $\mathrm{CH}_{4} /$ air reference flame on a matrix burner, and the LIF produced by this flame was used for online monitoring of the excitation wavelength. The remainder of the beam was expanded into a vertically collimated sheet, approximately $85 \mathrm{~mm}$ in height and $400 \mu \mathrm{m}$ in thickness, and transmitted to the combustor. Before reaching the combustor, a small portion of the incoming laser sheet was deflected into a quartz cell filled with a fluorescent dye solution, the signal from which was used to correct the OH-PLIF images for shot-to-shot sheet profile inhomogeneities. In experiment E1, the dye fluorescence was imaged into the same camera as the OH-PLIF images, while in E2, a second identical camera/lens/filter system was required due to geometrical restrictions. Fluorescence emitted by the OH radical was captured using an ICCD camera (LaVision Image Intense with I/I IRO) equipped with an achromatic UV lens (Nikkor, $f=105 \mathrm{~mm}$ at $f / 4.5)$. A UG11 and bandpass $(295-340 \mathrm{~nm}$ ) interference filter combination was used to isolated the OH-LIF signals from laser scattering and flame luminosity. In all cases, fluorescence was captured over a $400 \mathrm{~ns}$ intensifier gate. The images were corrected for the mean flame luminosity and camera whitefield sensitivity, but not for laser power absorption through the flame.

The PIV system consisted of a double pulsed, frequency doubled Nd:YAG laser (New Wave Research, Solo PIV) and either two (E1) or one (E2) CCD cameras (LaVision Imager Intense). The laser pulse pairs (time between pulses varying between 1-2 $\mu$ s depending on $u_{j}$ ) were expanded into a sheet, combined with the UV laser beam from the PLIF system using a dichroic mirror, and sent into the test section along the same beam path. Both the jet and crossflow were seeded with $\mathrm{TiO}_{2}$ particles having a mean diameter of approximately $1 \mu \mathrm{m}$. To avoid biasing the velocity calculations, seed levels were adjusted manually such that the density in both streams was approximately equal.

For the three-component S-PIV system used in E1, two cameras were mounted on Scheimpflug adapters and arranged in the angular configuration shown in Fig. 1 at angles of approximately $35^{\circ}$. Particle scattered light was collected into the cameras using commercial lenses (Nikkor, $f=50 \mathrm{~mm}$ at $f / 8$ ) equipped with bandpass and polarizing filters. Standard corrections were made for image defocusing and perspective distortion using Scheimpflug adapters and a 3D dot target (LaVision Type 7). The same target images were used to align the fields-of-view from the S-PIV cameras with that from the PLIF camera. Vector fields were computed from the particle image spatial cross-correlation using the LaVision DaVis 7.2 software package, with a 
final interrogation box size of 16 pixels. With a $50 \%$ interrogation box overlap, the spatial resolution and vector spacing were approximately $0.88 \mathrm{~mm}$ and $0.44 \mathrm{~mm}$ respectively.

The two-component vector fields in the $x-z$ planes measured during E2 were determined in a similar manner, but using a single camera configuration. The resolution was approximately the same as the S-PIV system.

Timing between the laser pulses and camera exposures was controlled using a pulse delay generator (BNC, Model 555) and a programmable timing unit (LaVision PTU). The simultaneous OH-PLIF and PIV measurements were performed at a framing rate of $4.9 \mathrm{~Hz}$, and a minimum of 400 frames was collected at each plane and condition. The OH-PLIF images were captured in between the two PIV laser pulses.

Laser Raman scattering was used to detect the mole fractions of the major species, from which the temperature $(T)$ and mixture fraction $(f)$ could be determined. The system consisted of a flashlamp-pumped dye laser (Candela LFDL 20, wavelength $=489 \mathrm{~nm}$, pulse energy $=3 \mathrm{~J}$, pulse duration $=3 \mu \mathrm{s})$, an achromatic lens $(D=80 \mathrm{~mm}$, focal length $=160 \mathrm{~mm})$, a spectrograph (SPEX 1802, focal length $=1 \mathrm{~m}$, slit width $=2 \mathrm{~mm}$, dispersion $\approx 0.5 \mathrm{~nm} / \mathrm{mm}$ ), and photomultiplier tubes (PMTs). The laser radiation was used to excite the vibrational Raman bands of $\mathrm{O}_{2}, \mathrm{~N}_{2}, \mathrm{H}_{2}$, and $\mathrm{H}_{2} \mathrm{O}$. Scattered light from the measurement volume (length and diameter $\approx 0.6 \mathrm{~mm}$ ) was collected at $90^{\circ}$ by the achromatic lens, relayed to the entrance slit of the spectrograph, and, after spectral separation, detected with single-shot resolution by the PMTs. The species number densities were calculated from these signals using calibration measurements and the temperature was deduced from the total number density using the ideal gas law. Simultaneous detection of all major species with each laser pulse also allowed determination of the instantaneous mixture fraction using the formulation of Bilger et al. [17].

Two hundred single shot Raman scattering measurements were taken at each of 147 points in the $z=0 \mathrm{~mm}$ plane for Case $3\left(u_{j}=200 \mathrm{~m} / \mathrm{s}\right)$. Due to laser reflections, accurate measurements were not obtained in close proximity to the injector plate; the measurements covered the range $x=-10-30 \mathrm{~mm}, y=6-30 \mathrm{~mm}$. More details of the Raman spectroscopy system, including a detailed error analysis, can be found in Meier et al. [18]. In the present experiment, a moderate amount of noise from scattered laser light and laser-induced luminescence from the quartz windows was detected, resulting in a mean offset in the $\mathrm{H}_{2}$ signal. This offset was corrected for, but increased the uncertainty of the derived quantities. Particularly significant for the results presented here are the expected uncertainties in the single-shot $\mathrm{H}_{2}$ mole fraction (approx. 5-10\%), temperature (approx. 8-10\%), and mixture fraction (approx. 10\%), as well as the mean temperature (up to approx. 6-8\%). It is noted that recorded signals were spatially averaged over the probe volume. Hence, a single measurement could contain fluid at different states, particularly in the vicinity of thin flame fronts.

\section{Results}

Figure 2 shows an instantaneous $\mathrm{OH}$ field overlaid with the simultaneous velocity streaklines in the $x-y$ plane at $z=0$ mm for the $200 \mathrm{~m} / \mathrm{s}$ jet. The flame at this instant is in a geometrically simple configuration, which visually resembles a lifted jet diffusion flame that is bent over by the cross-flow. There are two branches of the flame, which meet at a location approximately $25 \mathrm{~mm}$ downstream of the jet and $15 \mathrm{~mm}$ above the injection plane. The lower branch of the flame is stabilized directly in the lee of the jet and is lifted by a few millimeters from the combustor wall. This branch of the flame will be referred to as lee-stabilized, which is preferred over 'attached' (it is slightly lifted) or 'wake-stabilized' (wake can refer to the downstream jet-wake). The lee-stabilized flame branch had a very steady position, which will be discussed in Sec. 3.1. The upper branch of the flame, referred to as the lifted branch, was detached from any geometric features or coherent flow structures. The behavior 


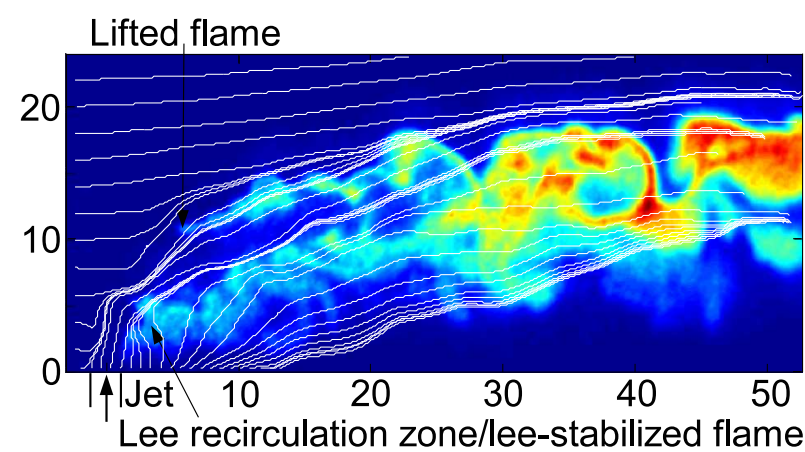

Fig. 2: Typical instantaneous $\mathrm{OH}$ field and velocity streaklines in the $z=0 \mathrm{~mm} x-y$ plane for Case $3\left(u_{j}=200 \mathrm{~m} / \mathrm{s}\right)$. Axis units are $\mathrm{mm}$.

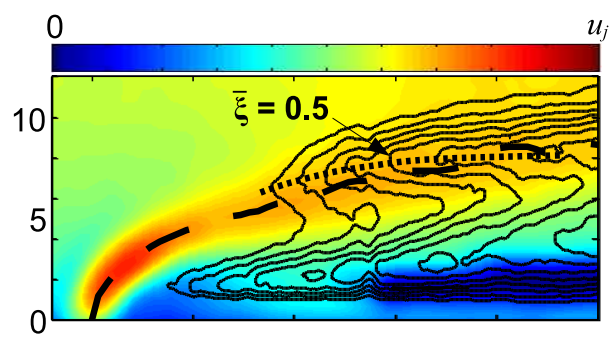

(a) Case $1-u_{j}=100 \mathrm{~m} / \mathrm{s}$

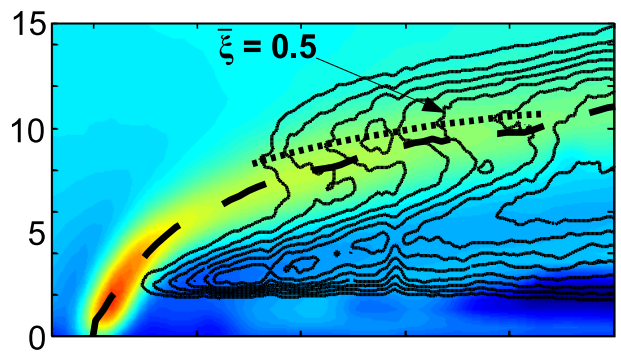

(b) Case $2-u_{j}=150 \mathrm{~m} / \mathrm{s}$

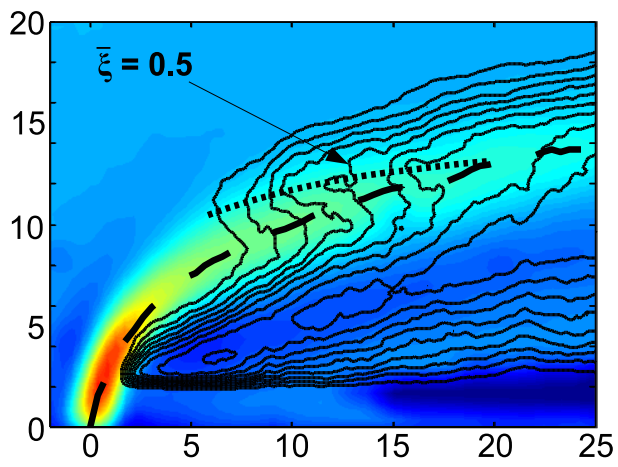

(c) Case $3-u_{j}=200 \mathrm{~m} / \mathrm{s}$

Fig. 3: Mean flow field and flame shape at the $z=0 \mathrm{~mm}$ plane for each case. Background is the velocity magnitude, dashed line is the jet trajectory $\left(y_{j}(x)\right)$, and solid lines are contours of $\bar{\xi}$ from 0.1-0.9 in 0.1 increments. The dotted line connects the leading edges of the $\xi$ contours at the lifted branch of the flame.

of this branch will be described in Sec. 3.2. It is noted that, unlike a conventional jet flame, the JICF has a high degree of asymmetry about the jet trajectory. It also should be noted that these descriptions are based on planar measurements of three-dimensional structures. Caution must therefore be taken when interpreting the images. The presented results are consistent with the data from the $z \neq 0 \mathrm{~mm}$ and transverse measurement planes.

The OH PLIF signal shown in Fig. 2 not only indicates heat-release regions, but also all regions containing hot products 
above approximately $1500 \mathrm{~K}$. Ambiguities therefore exist in associating the $\mathrm{OH}$ signal with flame structures, particularly at downstream locations. However, upstream transitions between low and high $\mathrm{OH}$ signal must occur at a reaction zone, and the $\mathrm{OH}$ boundaries at the most upstream edges of the flame branches represent reacting flame structures. Furthermore, the $\mathrm{OH}$ layer boundary facing inwardly towards the jet fluid represents the location of diffusion-flame reactions (c.f. Donbar et al. [19]).

Major statistical flame and flow features are illustrated in Fig. 3, which shows the mean velocity field magnitude, jet trajectory, and a flame position indicator for each case studied. The jet trajectories, $y_{j}(x)$, are defined as the path of maximum velocity magnitude starting from the jet exit. Experimental and computationally derived scaling relationships for non-reacting JICF trajectories with high momentum flux ratios $(r \gg 1)$ generally take the form [1]

$$
\frac{y_{j}}{r d}=A\left(\frac{x}{r d}\right)^{B}
$$

where $A$ is a coefficient representing the rate of cross-flow fluid entrainment and $B$ is a shape constant. Various values for $A$ and $B$ have been proposed for different ranges of $r$ and different jet exit velocity profiles $[1,20]$. For $5 \leq r \leq 25$, similarity analysis in non-reacting flows indicates that $B=1 / 2$ and $1 / 3$ in the jet near- and far-fields, respectively, while experimental results have been reported in the range of $0.25<B<0.34$ and $1.2<A<2.6$ [21]. While the arguments used to derive Eq. 2 and its parameters are strictly valid only for non-reacting configurations with $r \gg 1$, the jet trajectories measured for the reacting, $r \approx 1$ jets studied here fit very well to this general form. Based on a least-squares regression, the fit constants were found to be in the ranges $1.2<A<1.3$ and $0.42<B<0.43$ for all cases.

To visualize the statistical position of the flame, the OH PLIF images were converted to binary, with a value of $\xi=0$ and 1 in low and high $\mathrm{OH}$ regions, respectively. The threshold level was selected such that visually apparent flame structures at the leading edge of the lifted branch were above the cutoff (e.g. lifted and lee-stabilized branches in Fig. 2). A uniform signal level was used for all data, and the results are reasonably independent of the threshold; a $20 \%$ change in threshold level results in an approximately $5 \%$ change in the position of the $\bar{\xi}$ contours. The ensemble average, $\bar{\xi}$, then gives information about the statistical transition between cold reactants and hot products. $\bar{\xi}$ is analogous to the mean reaction progress variable field in premixed combustion. However, similar to the instantaneous OH PLIF images, interpretation of $\bar{\xi}$ contours as representing the statistical reaction zones is limited; $\bar{\xi}$ represents the flame position only at the leading edge contours and contours facing the jet. It is noted that $\bar{\xi}$ provides considerably more information than an ensemble average of the OH PLIF directly, since the actual $\mathrm{OH}$ signal at the flame leading edge is low relative to the signal in the products downstream of the flame tip.

All cases exhibit the general structure that was seen in Fig. 2, containing lee-stabilized and lifted flame branches. The distance between $\bar{\xi}$ contours is indicative of the positional steadiness of the flame branch, with tightly spaced contours indicating a steadier flame. As can be seen, the lee-stabilized branch was very steady in the $u_{j}=200 \mathrm{~m} / \mathrm{s}$ case, and became more unsteady with decreasing jet velocity. The lifted branch was highly unsteady in all cases. The behavior of these flame branches is discussed more below.

Figure 4 shows the temperature field measured in the vicinity of the jet for Case $3\left(u_{j}=200 \mathrm{~m} / \mathrm{s}\right)$ from the Raman scattering. As can be seen, there are two local temperature maxima, corresponding to the lee-stabilized and lifted flame branches. 


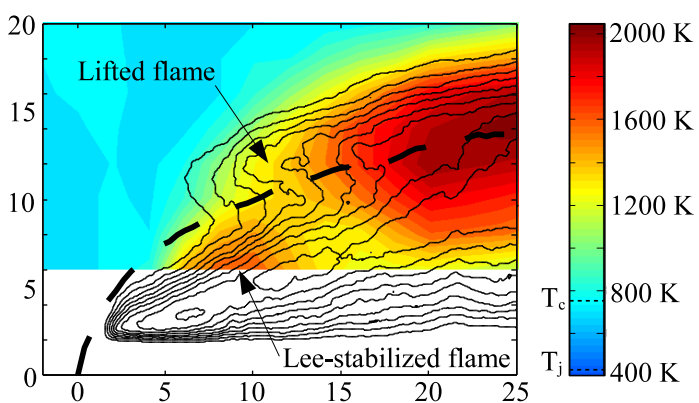

Fig. 4: Temperature field measured using Raman scattering with the $\bar{\xi}$ contours in the vicinity of the jet for Case $3\left(u_{j}=200 \mathrm{~m} / \mathrm{s}\right)$. Jet trajectory is shown as the dashed line.
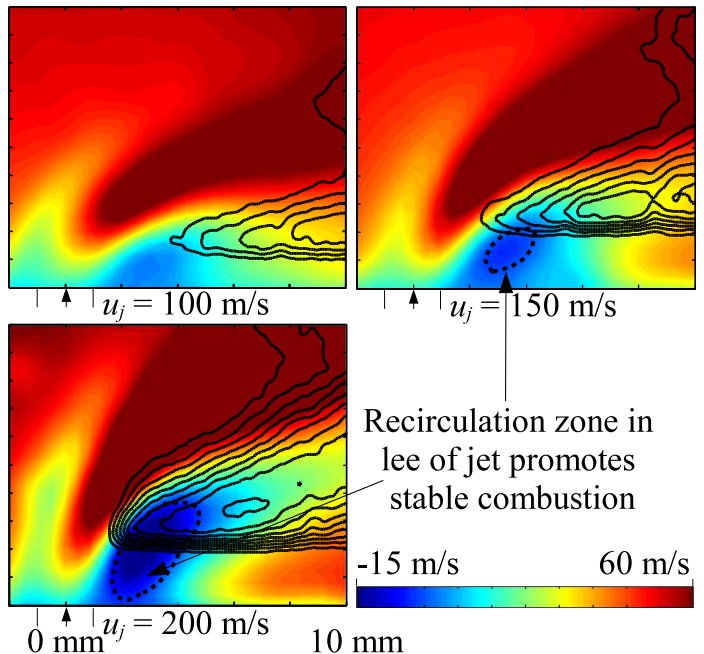

Fig. 5: Mean axial velocity fields $\left(\bar{u}_{x}\right)$ in the vicinity of the jet for all cases. Dotted lines are the regions in which $\bar{u}_{x}<0$. Solid lines are contours of $\bar{\xi}$ from $0.1-0.9$ in 0.1 increments.

The leading edge of lee-stabilized branch was at approximately $y=2-4 \mathrm{~mm}$, which is in agreement with the DNS results of Grout et al [8]. Particularly, the $\bar{\xi}$ contours in the lee-stabilized branch for the $u_{j}=200 \mathrm{~m} / \mathrm{s}$ jet show similar features to the mean heat release contours in the corresponding region of the DNS flame (which had a $250 \mathrm{~m} / \mathrm{s}$ square jet).

The positional stability of the lee branch varied with the jet velocity. This is indicated by the spacing of the $\bar{\xi}$ contours, with closer contours indicating a stable position. At the $u_{j}=200 \mathrm{~m} / \mathrm{s}$ condition, the lee-stabilized flame branch had a very steady axial position, approximately $2 \mathrm{~mm}$ downstream of the jet. As the jet velocity decreased, the instantaneous position at which the flame anchored became increasingly unsteady. This increasing unsteadiness is due to a weaker, smaller, and less stable low-velocity region in the lee of lower momentum jets. Figure 5 shows the mean axial velocity field and $\bar{\xi}$ contours at each condition in the vicinity of the jet exit. At $u_{j}=200 \mathrm{~m} / \mathrm{s}$, a large region of negative axial velocity forms in the jet lee. The strength (i.e. negative axial velocity magnitude) and size of this region decrease as the jet momentum decreases, until no mean recirculation zone is observed at $u_{j}=100 \mathrm{~m} / \mathrm{s}$. Hasselbrink and Mungal [21] showed that the recirculation is due to the cross-flow wrapping around the perceived obstruction caused by the jet, which results in an opposed-flow configuration at the symmetry plane in the jet lee. A perceived source therefore exists at the symmetry plane, with the strength of the source increasing with the jet momentum. The associated low speed region provides a stable position in which the lee-stabilized flame branch can anchor.

\subsection{Lifted flame branch}

The lifted flame branch in Fig. 2 visually resembles a conyentional lifted jet flame. While lifted branch JICF flames are 

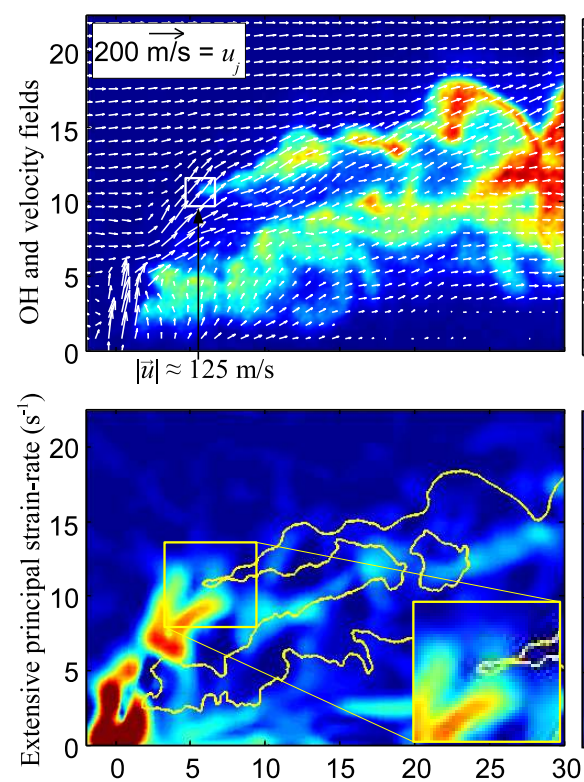

(a) Straight-edge lifted branch

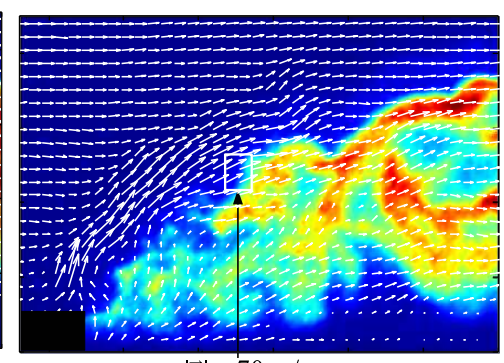

$|\vec{u}| \approx 70 \mathrm{~m} / \mathrm{s}$

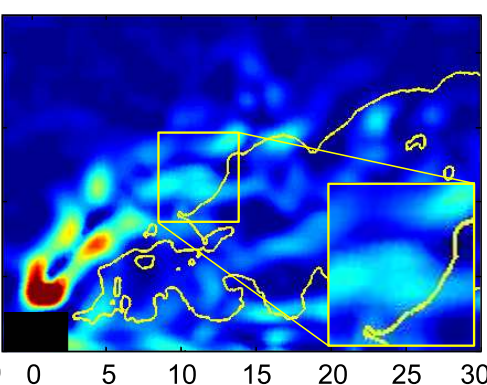

(b) Merged lifted branch

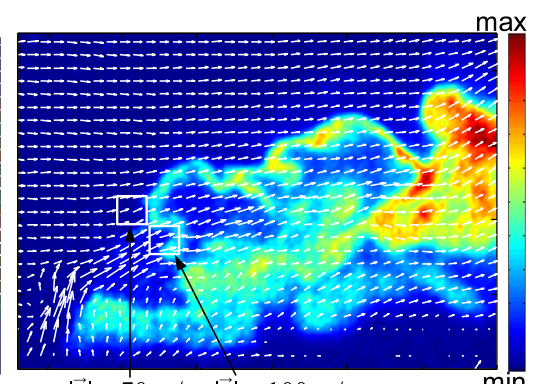

$|\vec{u}| \approx 70 \mathrm{~m} / \mathrm{s} \quad|\vec{u}| \approx 100 \mathrm{~m} / \mathrm{s}$

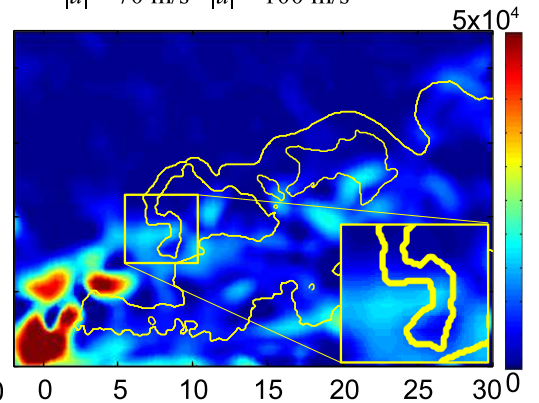

(c) Hooked edge lifted branch

Fig. 6: Typical flames and flow-fields showing different lifted-branch configurations. Images are from the $z=0 \mathrm{~mm}, x-y$ plane in the $u_{j}=200 \mathrm{~m} / \mathrm{s}$ jet. Note that the color scale for the strain-rate figures is different than in Fig. 7 .

more complex than conventional flames due to the flow, mixing, and combustion asymmetries, they do have some similar features. Lyons [22] identifies five classical theories for stabilization of lifted jet flames, the majority of which involve the propagation of the upstream flame boundary into the oncoming fluid through either (partially) premixed flame or tribrachial flame propagation. This propagation may be enhanced by large-scale turbulent structures. Furthermore, while a theory based on flame extinction has largely been superseded, the effects of fluid mechanics on the local reaction rate can be important. These theories are not mutually exclusive; the same system may undergo tribrachial- and/or premixed flame propagation that is enhanced by eddy transport and have a propagation speed that is affected by the local strain-rate field. It is noted that the tribrachial flame structure is an edge flame composed of lean and rich premixed flame wings, with a trailing diffusion flame, whereas partially premixed flames propagate into regions of stratified, but flammable fuel/air mixtures.

Three common lifted branch geometries observed in the $x-y$ plane at $z=0$, designated as straight, merged, and hooked are shown in Fig. 6 from the $u_{j}=200 \mathrm{~m} / \mathrm{s}$ jet. Similar structures were found at the other jet velocities. Each flow field and flame is visualized by the velocity vectors with the OH PLIF field, and by the principal extensive strain-rate field with the boundary of the thresholded OH PLIF field. The threshold used to determine this boundary contour was the same as the threshold used to compute $\xi$. The principal extensive strain-rate is the largest eigenvalue of the rate-of-strain tensor, $S_{i j}=1 / 2\left(\partial u_{i} / \partial x_{j}+\partial u_{j} / \partial x_{i}\right)$, and indicates regions of high extensive strain-rate in the local coordinates of the flow field. Based on the planar PIV measurements, only the in-plane strain-rates could be calculated. Hence, the $x-y$ plane images show the principal extensive strain-rate in this plane, designated $\zeta_{x y}$. Figure 7 shows two representative measurements in the $x-z$ plane at $y=12 \mathrm{~mm}$ from the $u_{j}=200 \mathrm{~m} / \mathrm{s}$ jet. This $y$-value is approximately at the statistical height of the lifted branch leading edge for this case (c.f. Fig. 3c). The principal strain-rate calculated from the $x-z$ plane is $\zeta_{x z}$. For reference, Fig. 7 also show the location at which the mean jet trajectory crosses $y=12 \mathrm{~mm}$.

It is noted that interpretation of the $\mathrm{OH}$ images from the $y=12 \mathrm{~mm} x-z$ plane (Fig. 7 ) is complicated by the fact that 


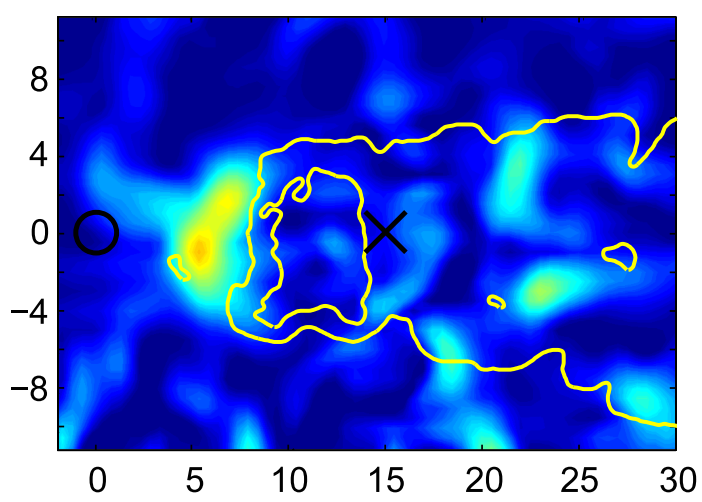

(a) Straight-edge lifted branch

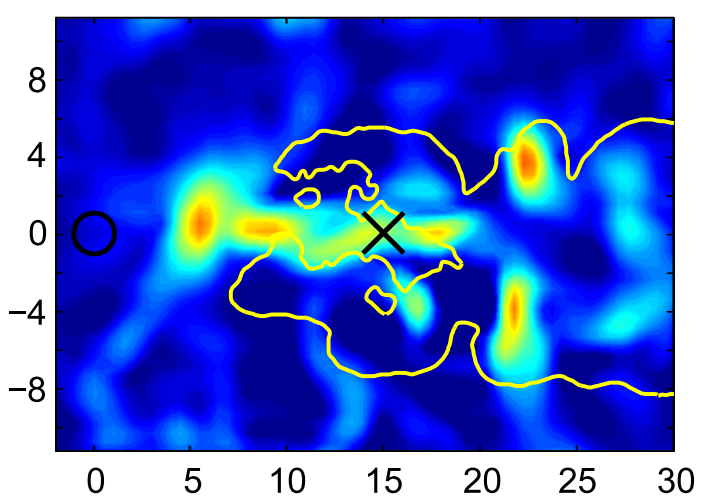

(b) Merged lifted branch

Fig. 7: Typical extensive principal strain-rate fields $\left(\zeta_{x z}\right)$ with OH layer topographies in the $y=12 \mathrm{~mm}, x-z$ plane in the $u_{j}=200 \mathrm{~m} / \mathrm{s}$ jet. $\circ$ is the jet exit and $\times$ is the location where the mean jet trajectory crosses the plane. The color map is the same as that in Fig. 6 , but the scale on the strain rate is $0-2.5 \times 10^{2} \mathrm{~s}^{-1}$.

the wrinkled $\mathrm{OH}$ layers lie at an acute angle to this plane, and therefore cross it in a complex manner. The most upstream position of the flame along the centerline in Fig. 7a agrees well with the most upstream positions of the straight geometry Fig. 6a. The $\mathrm{OH}$ region then exits the plane, before reappearing downstream of the point where the lifted and lee-stabilized branches merge. The thin horizontal $\mathrm{OH}$ layers between $x \approx 5-15 \mathrm{~mm}$ represent the continuous flame surface connecting the upper lifted branch to the lower lee-stabilized branch. From the location of the mean jet trajectory relative to these features, it is apparent that the flame envelopes the jet, which is similar to the flame geometry observed in the instantaneous volume rendering of the DNS temperature field by Grout et al [8]. The configuration in Fig. 7b is analogous to Fig. $6 \mathrm{~b}$ and will be discussed more below.

The straight geometry shown in Fig. 6a resembles the structure observed at the base of conventional lifted jet flames. Numerous instantaneous measurements with similar geometries were found for all jet cases. There is a clear relationship between the extensive strain-rate field and the leading edge of the lifted-branch in both this image and Fig. 7; the flame resides immediately downstream of a high strain-rate region.

Figure 8 shows a scatter plot of the temperature versus mixture fraction at locations in the vicinity of the lifted branch leading edge in Fig. 6a, determined from the Raman measurements. Also plotted are equilibrium results from unstrained and strained flame calculations, which were determined using Cantera [23]. The two strained flame calculations shown correspond to the curve best enveloping the data $\left(a_{t}=3000 \mathrm{~s}^{-1}\right)$ and at the mean extensive principal strain-rate at $(x, y)=(6,10)$ mm $\left(a_{t}=15000 \mathrm{~s}^{-1}\right)$. The high strain-rates in this region have a very clear effect on the combustion. All data points at 


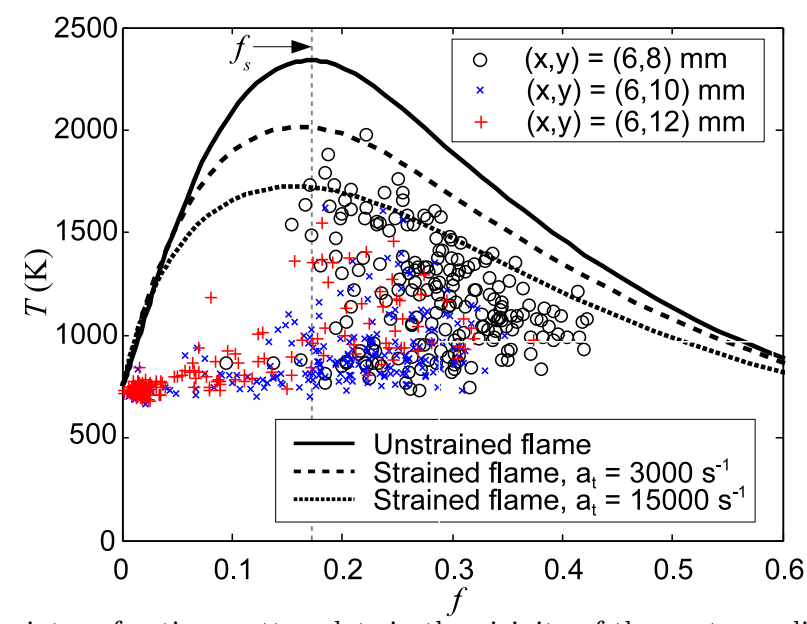

Fig. 8: Temperature versus mixture fraction scatter plots in the vicinity of the upstream lifted flame branch in Fig. 7 a.

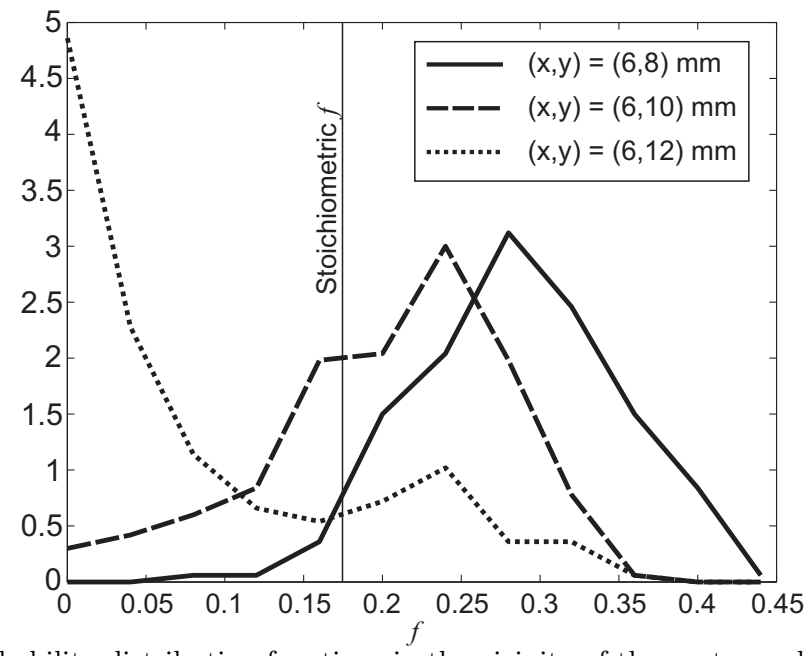

Fig. 9: Mixture fraction probability distribution functions in the vicinity of the upstream lifted flame branch in Fig. 7a.

$(x, y)=(6,10)$ and $(6,12)$ fell below the calculated curve at the mean strain-rate, and few points at $(x, y)=(6,8)$ were above the curve. This indicates that the local strain-rate is important in determining the combustion properties in this region, as was indicated by the flow field images. The moderate temperature mixtures, well below the equilibrium combustion calculation, are evidence of of intermediate reaction states (hot products mixed with fresh gas without reaction), local flame extinction, or spatial averaging over the probe volume.

The scatter plots show that there was a large number of unreacted samples on both the fuel-lean and rich sides of stoichiometric $\left(f_{s}=0.17\right)$. As expected, more rich and lean samples were found at lower and higher $y$ locations, respectively. Figure 9 shows mixture fraction probability density functions (PDF) at the same locations as the scatter plots. Predominantly fuel-rich samples were found at $(x, y)=(6,8) \mathrm{mm}$ and mostly pure air samples were found at $(x, y)=(6,12) \mathrm{mm}$. This high degree of unmixedness indicates that combustion in this region exists predominantly as tribrachial flames.

Another commonly occurring flame configuration in the $x-y$ plane at $z=0$ is the merged configuration shown in Fig. $6 \mathrm{~b}$, wherein the lifted flame branch has either been fully extinguished or lifted sufficiently far that it merged with the downstream products from the lee-stabilized branch. Similar to the straight configuration, the flame in this merged configuration is downstream of a high strain-rate region. Figure $7 \mathrm{~b}$ shows an $x-z$ plane image with a similar flame phenomenon. In this image, a region of high strain-rate extends axially along the centerline to about $x=20 \mathrm{~mm}$, and the leading edge of the flame 


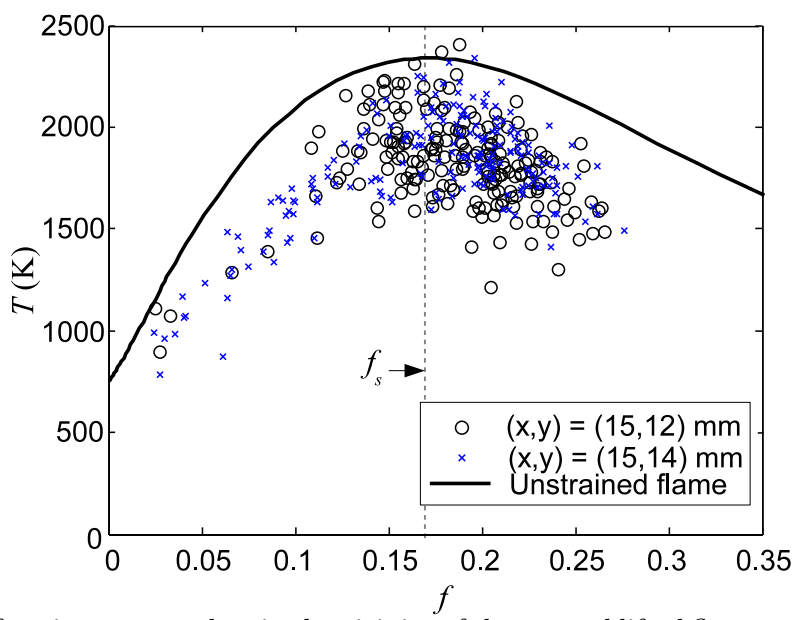

Fig. 10: Temperature versus mixture fraction scatter plots in the vicinity of the merged lifted flame configuration for Case $3\left(u_{j}=200 \mathrm{~m} / \mathrm{s}\right)$

at $z=0 \mathrm{~mm}$ resides immediately downstream of the strain-rate. Hence, it appears that the fluctuating strain-rate along the jet centerline is sufficient to occasionally cause local extinction of the lifted branch.

Figure 10 shows scatter plots of temperature versus mixture fraction at locations in the vicinity of the merged flame branch. This region contained a wide variety of states. Several samples were at the burnt gas conditions of an unstrained flame, whereas many other samples were at relatively low temperatures. Few samples showed very lean or very rich mixtures, and many instantaneous measurements showed mixtures of unburnt hydrogen and oxygen across a range of flammable mixture fractions. Hence, the flame propagation in this region is generally through a stratified mixture of fuel, oxidizer, and products.

The present measurements show several similarities to the DNS of Grout et al [8] and Kolla et al. [9]. However, one major difference is the position and shape of the lifted branch. In the DNS results, the lifted branch was statistically attached to the lee-stabilized branch near the leading edge, crossing the jet flow a few millimeters downstream of jet exit. In the present measurements, the two branches generally were detached. Neither the PLIF nor Raman measurements show indications of the lifted and lee-stabilized branches being connected near the leading edge. However, the lifted branch would occasionally cross the jet flow to connect with the lee-stabilized branch farther downstream. These connections generally exhibited the hooked geometry shown in Fig. 6c and occurred downstream of the high strain-rate regions. The differences between the experiments and DNS therefore may be related to the method used to simulate the effects of the local flow field on the reaction rate or slight differences in properties (e.g. boundary layer thickness) between the simulation and experiment. It is noted that the occurrence of the hooked geometry was not associated with large vortical structures aligned perpendicular to the $x-y$ plane. It may be possible that flames with this geometry are the result of vertical wake-vorticies, however this could not be confirmed from the present measurements.

\section{Conclusion}

Fluid mechanical and mixture properties that influence the structure and stabilization of jet flames in cross-flows were experimentally investigated using a variety of laser diagnostic techniques. In all cases, the flame in the $z=0 \mathrm{~mm}$ plane consisted of two branches, one stabilized in the lee of the jet and one lifted along the upper side of the jet. These branches were connected by a flame that enveloped the jet trajectory in three dimensions. The jet trajectory followed classical scaling laws.

The position of the lee-stabilized flame branch was more stable in the higher momentum jets due to a stronger recirculation 
zone in the jet-lee. The lifted flame branch exhibited considerable dynamics, sometimes stabilizing relatively close to the jet and sometimes being pushed back all the way to the flame tip. The position of the flame was found t0 depend strongly of the position and shape of the extensive principal fluid-dynamic strain-rate field. A wide variety of mixture fractions, from very lean to very rich, were present at flame-stabilization locations near the jet. At flame stabilization locations further downstream,

well mixed flammable regions of combustion products with unburnt air and fuel often were found. This indicates that the structure and stabilization of the lifted branch is influenced by both tribrachial and stratified premixed flame behaviors.

\section{References}

[1] A. R. Karagozian, Prog. Energy Combust. Sci. 36 (2010) 531-553.

[2] P. J. Vermeulen, P. Grabinski, V. Ramesh, J. Eng. Gas Turb. Power 114 (1992) 46-54.

[3] J. D. Holdeman, Prog. Energy Combust. Sci. 19 (1993) 31-70.

[4] J. Fleck, P. Griebel, A. M. Steinberg, M. Stöhr, M. Aigner, A. Ciani, J. Eng. Gas Turb. Power.

[5] J. S. Abbitt, C. Segal, J. C. McDaniel, R. H. Krauss, R. B. Whitehurst, J. Prop. Power 9 (1993) $472-478$.

[6] M. Gruber, J. Donbar, C. Carter, K.-Y. Hsu, J. Prop. Power 20 (2004) 769-778.

[7] D. J. Micka, J. F. Driscoll, Combust. Flame 159 (2012) 1205-1214.

[8] R. Grout, A. Gruber, C. Yoo, J. Chen, Proc. Combust. Inst. 33 (2011) 1629-1637.

[9] H. Kolla, R. W. Grout, A. Gruber, J. H. Chen, Combust. Flame In Press.

[10] R. M. Kelso, A. J. Smits, Phys. Fluids 7 (1995) 153-158.

[11] S. H. Smith, M. G. Mungal, J. Fluid Mech. 357 (1998) 83-122.

[12] L. L. Yuan, R. L. Street, J. H. Ferziger, J. Fluid Mech. 379 (1999) 71-104.

[13] S. Muppidi, K. Mahesh, J. Fluid Mech. 574 (2007) 59-84.

[14] S. Muppidi, K. Mahesh, J. Fluid Mech. 598 (2008) 335-360.

[15] T. Fric, A. Roshko, J. F 279 (1994) 1-47.

[16] R. F. Huang, S. M. Wang, Combust. Flame 177 (1999) 56-77.

[17] S. S. R.W. Bilger, R. Kee, Combust. Flame 80 (1990) 135-149.

[18] W. Meier, P. Weigand, X. Duan, , R. Giezendanner-Thoben, Combust. Flame 150 (2007) 2-23.

[19] J. M. Donbar, J. F. Driscoll, C. D. Carter, Combust. Flame 122 (2000) 1-19.

[20] T. H. New, T. T. Lim, S. C. Luo, Ex 40 (2006) 869-875.

[21] E. Hasselbrink, M. G. Mungal, J. Fluid Mech. 443 (2001) 1-25.

[22] K. M. Lyons, Prog. Energy Combust. Sci. 33 (2007) 211-231.

[23] D. G. Goodwin, in: F. M. M. Allendorf, F. Teyssandier (Eds.), CVD XVI and EuroCVD Fourteen, Electrochemical Society, 2003, pp. 155-162. 